\title{
Participación social entre los adultos mayores chilenos
}

Predictors of social participation among chilean elderly

Ma Beatriz Fernández Lorca

Doctora en Sociología. Instituto de Sociología. Pontificia Universidad Católica de Chile.Correo electrónico:mrfernan@uc.d

\section{Resumen}

El presente artículo busca conocer los principales determinantes de la participación social entre los adultos mayores en Chile. Para ello se trabajó con los datos de la Primera Encuesta Nacional de Calidad de Vida en la Vejez del año 2007, con 1616 participantes. Se encontró, mediante la realización de una serie de regresiones logisticas, que una mayor educación, una adecuada funcionalidad y una percepción positiva sobre la vejez, son elementos que incrementan significativamente las posibilidades de participación entre las personas de 60 años y más.

Palabras clave. Participación social, adultos mayores, organizaciones y actividades sociales.

Abstract

This paper aims to know the main determinants of social participation among older adults in Chile. Using data from the First National Survey of Quality of Life in Old Age in 2007, it was found by performing a series of logistic regressions that higher levels of education, an adequate function tion among people aged 60 and older.

Key words. Social participation, older people, social organizations and activities.

\section{Introducción}

El objetivo del presente artículo es analizar las principales características de la participación de los adultos mayores en diversas asociaciones o agrupaciones sociales a lo largo del país, estableciendo los principales determinantes que estarían mediando en dicho involucramiento. Esto se justifica en la medida en que la II Asamblea Mundial sobre Envejecimien enfatizó la los mayores, to como mames, tanto por sucontribucion a la sociedad como también a la calidad de vida de los mismos. En Chile, el grupo de 60 y más años ha tenido un aumento sostenible durante las últimas décadas, alcanzando en la actualidad un 13\% (con cerca de dos millones doscientos mil), a la vez que la esperanza de vida se ha incrementado notablemente, llegando a los 79 años en promedio para el periodo 20102015 (CEPAL, 2009), con lo cual la participación en la sociedad es un modelo de vejez necesario. Sin embargo, persiste una visión negativa en torno a la vejez, en la medida en que se percibe a la población mayor únicamente como receptora de pensiones y beneficiaria de diversas prestaciones sociales, olvi- dando las contribuciones que los propios mayores han realizado a lo largo de su vida y las que siguen realizando al ayudar a sus familiares, o bien al participar en actividades sociales de diferentes organizaciones, sin contraprestación económica alguna. Los adultos mayores no son un grupo homogéneo, caracterizado solamente por la dependencia y falta de autonomía, sino que cada vez son más las personas capaces de vivir la vejez con niveles de salud física y mental aceptables, lo cual les permite mantener su independencia, realizar actividades y aportar diversos recursos y servicios a su entorno (Engler 2005; Triadó, Celdrán, Conde y otros, 2008).

a participación de los mayores en la toma de decisiones de distintos ámbitos sociales y políticos es un proceso imprescindible, tanto por su ver epresentación adecuada en términos cuantitativos (IMSERSO, 2008). De este modo, muchas de las propuestas planteadas por diversos organismos instituciones han estado dirigidas a promover la participación real y efectiva de los adultos mayointegración en la vida 
enfoque de derechos, el cual aboga porque los individuos dejen de ser considerados solamente com objetos de asistencia y de cuidados, promoviéndose más bien un continuo empoderamiento de estos (CELADE, 2003).

Simultáneamente, la participación de los adultos mayores también contribuye a su propia calidad de vida, en la medida en que formar parte activa y comprometida de una actividad conjunta puede llevar a los sujetos a experimentar altos niveles de satisfacción como resultado de dicho involucramiento (González, 1994). Es así como diversos estudios han demostrando que aquellos adutos mayores que han demostrando que aquellos advos mayores que participan en grupos sociales mantienen una calidad de vida superior a los que están socialmente aislados, encontrándose además correlaciones positiva entre la percepción de bienestar y una vida activa (Beck y Page, 1988; Krzemien, 2001). Asimismo, McGuinn y Mosher (2000) muestran cómo las actividades que se realizan en colaboración con otros tienen más efectos positivos sobre el bienestar que aquellas que se incrementa si dicha actividad es una iniciativa del propio individuo. Igualmente se ha demostrado que altos niveles de participación social se asocian con menores niveles de suicidio, mejor salud física y menor mortalidad o mayor supervivencia de los individuos (Holt-Lunstad, Smith y Layton, 2010; House, Landis y Umberson, 1988; Lennartsson y Silve, Landis y Umberson, 1988; lennatsson Silverstein, 2001; Seeme , 2000), todo logual se condice con las ideas de Rowe y Kahn (1997), para quienes un compromiso activo y productivo con la sociedad es un componente central de una vejez exitosa.

Ahora bien, en cuanto al contenido de la participación social, Connidis y McMullin (1992) señalan que esta engloba una diversidad de actividades tales como ir a lugares públicos, viajar o hacer cualquier actividad fuera del hogar. Por su parte, McDonald (1996) considera más bien que la participación se asocia a tres dimensiones: vecindario, implicación en actividades organizadas y amistad. Con esto se observan dos tendencias, una mucho más flexible, y otra orientada a un plano más bien formal de participación.

En el caso específico de nuestro país, el Servicio Nacional del Adulto Mayor (2003) establece que la participación social se relaciona con la posibilidad que tiene el ciudadano para intervenir, ser tomado en cuenta e integrarse en la vida de su comunidad, lo cual se realiza a través de diversas organizaciones comunitarias, como son los clubes de adultos mayores, uniones comunales y juntas de vecinos.
No obstante, se destaca además que la participación social puede darse en organizaciones de otro tipo, como son las cooperativas, organizaciones no gubernamentales, programas para el adulto mayor en las municipalidades, organizaciones deportivas, ch las muni giosas, etc. Con ello, queda establecido que la participación social puede desarrollarse en diversos planos sociales, que finalmente le reportarán a los adultos mayores una serie de beneficios sobre su bienestar y calidad de vida. Sin embargo, el involucramiento activo y comprometido en alouna actividad organizada y decarrolluda comidad por parte del adulto yeyor to mayor dependerá finalmente de diversos factores que pueden incentivar o bien reducir dicha participación, y que son analizados con más detalle a continuación.

\section{Factores asociados a la participación}

\section{social de los adultos mayores}

Con respecto a los posibles factores que puedan estar mediando en la participación social de los mayores, la evidencia sugiere en primer lugar que las mujeres participan más que los hombres, porque en su gran mayoría están viudas -dada la mayor esperanza de vida que poseen en comparación con los hombres- y sus familias tienden a tener un ciclo de vida avanzado, con lo cual la participación es una importante vía para establecer nuevas relaciones sociales y evitar así la soledad.

Del mismo modo se señalan diferencias según la edad. Griffin y McKenna (1998) observaron que el nivel de participación y actividad se reduce a medida que aumenta la edad y existen peores niveles de salud. Con ello se suele afirmar que el estado de salud o el nivel de capacidad funcional puede condicionar el tipo y cantidad de actividad en la cual se implica una persona; los mayores más sanos podrían estar mejor preparados para asumir diversos roles e implicarse en más actividades y redes de interacción social. Lefranchois, Leclerc y Poulin (1998) han encontrado que el estado de salud es uno de los principales obstáculos para el desarrollo de actividades y de participación en la vejez, siendo incluso este mayor que el impacto de la edad en sí misma.

Otro elemento a considerar es la educación. Una posible explicación es que las personas mayores con un alto nivel educativo tendrían una mejor salud que aquellas con menores niveles educativos, lo que puede facilitar la participación (Dávila de León y Díaz Morales, 2009). Igualmente este factor ha mostrado tener un efecto sobre la calidad de vida (Barros, Forttes y Avendaño, 2004; Barros, Forttes y Herrera, 2006), mediante el acceso a conocimientos, hábitos de vida y mejores oportunidades de ingreso. La adquisición de un buen nivel educacional constituye así una ventaja acumulativa que se acrecienta con el pasar de los años, lo cual puede favorecer un mayor involucramiento social de las personas durante la vejez.

Otro aspecto asociado, mencionado por Vega y otros (2002), es la autopercepción de la vejez por parte de los mayores: aquellos con una mala imagen o percepción negativa de esta etapa, probablemente no estarán motivados y no querrán participar mientras sí lo harán quienes denes son optimistas y perciben a esta etapa como una nueva oportunidad para realizarse e involucrarse con el entorno. Por último, se menciona que las características del entorno social y las relaciones familiares también pueden afectar la participación de los mayores. No obstante la evidencia no es concluyente. Algunos autores señalan que tener buenas relaciones sociales puede favorecer la participación social (Warburton, Ferry, Rosenman y otros, 2001), mientras que otros señalan que no existiría relación (Warburton y Stirling, 2007).

\section{Metodología}

\section{Características de la muestra}

Los datos que se analizan proceden de la Primera Encuesta Nacional de Calidad de Vida en la Vejez, realizada el año 2007 en nuestro país por el Instituto de Sociología de la Pontificia Universidad Católica de Chile, y financiada por la Caja de Compensación Los Andes. Esta encuesta es representativa del $75 \%$ de la población de 60 años y más a nivel nacional, residente en viviendas particulares en ciudades de más de 30.000 habitantes. Se aplicó un cuestionario estructurado cara a cara a una muestra aleatoria probabilística de 1616 casos.

\section{Estimación modelo}

Como se ha señalado, el propósito de este artículo es poder establecer los principales factores que se relacionan con la participaci tos mayores en nuestro país. Para ello se utiliza la técnica de regresión logística, que es la más común para trabajar con datos binarios (Agresti, 2007), la cual básicamente permite predecir o estimar la probabilidad de ocurrencia de un suceso -en este caso participar (o no) en diversas organizaciones y actividades sociales-, en función de un conjunto de predictores con capacidad explicativa respecto a la variable dependiente.
Variables dependientes: participación social

Para la presente ocasión, la participación social activa y comprometida se define operacionalmente como la participación una o más veces al mes en las siguientes organizaciones y actividades: clubes, voluntariado, actividades religiosas y en programas dirigidos hacia los adultos mayores (específicamente talleres). Según los datos de la encuesta, un 58\% de los mayores a lo largo del país participa con cierta intensidad en dichas agrupaciones sociales. De manera específica, un $47 \%$ del total de los adulto mayores señala participar en algún movimiento religioso, el 26\% señala que asiste algún club, un 12\% en voluntariado y un $11 \%$ en diversos talleres.

Posteriormente, se crearon cuatro variables a partir de las preguntas sobre si la persona mayor participaba, y con qué intensidad, en clubes, actividades religiosas, voluntariado y talleres. Se agruparon las categorías de una vez al mes y más en sí participa, y el resto en no participa, para todos los casos. Adicionalmente se construyó una única variable de participación, a partir de cada una de las nuevas variables dicotomizadas; si al menos en uno de los 4 casos la persona participaba se le asignó el valor $1=$ participa, $y$ de lo contrario, $0=$ no participa.

\section{Variables independientes}

- Características sociodemográficas:

Género: $0=$ Hombre y $1=$ Mujer:

Edad: $0=60$ a 74 años y $1=75$ y más años;

Vive con pareja: $0=$ No y $1=\mathrm{Si}$

Región: 0=Otras regiones y l= Región Metropolitana.

- Educación: se agrupó esta variable en $0=$ Edu cación básica o menos y l= Educación media y más.

- Capacidad funcional: este índice combina la capacidad de las personas para realizar ciertas actividades de la vida diaria, como son caminar 100 mts., subir más de un piso, manejar dinero, calentar el agua para el café o el té y apagar la cocina, administrar o manejar sus propios medicamentos, andar en micro o metro, con las siguientes alternativas: 1. Puede o podría hacerlo sin dificultad. 2. Puede o podría hacerlo, pero con dificultad, 3. No puede hacero. Es relevancon dificultad, 3. No puede hacerlo. Es relevane destacar que las tareas que se han evaluado intentan evitar el sesgo cultural y de género, a incluir tareas que tanto hombres como mujeres son capaces de realizar, y a su vez excluir otras que los sujetos no han hecho nunca por razones más bien socioculturales como es, por ejemplo, 
que en algunos países de de Latinoamérica es poco frecuente que los hombres cocinen, o hay mujeres que no manejan asuntos económicos.

El índice se comporta de manera unidimensional, con un alfa de Cronbach de 0.844. Se sumaron las seis variables, de lo que resulta un rango de variación de 6 a 18. Al explorar qué divisiones dicotómicas de este índice se relacionaban de manera más clara con participación se decidió dicotomizar el índice en: $\mathrm{O}=$ con dificultades (vadicotom 7 o más) y le con buen funcionamiento lores 7 o más) y (valores 6-7)

- Condiciones de salud: este índice combina la presencia del tratamiento de enfermedades dicotomizado en: sin enfermedades/una dos o mis en tratamiento $y$ al menos una enfermedad sin tratamiento y la percepción de salud, dicotomizada en buena y regular/mala. Resultan cuatro combinaciones posibles, desde mal en aspectos objetivos y subjetivos, mal en uno y bien en otro ( $y$ viceversa), hasta bien en ambos, que finalmente fueron recodificadas en $0=$ Condiciones de salud poco adecuadas (condiciones objetivas y evaluán subjetiva mal) y l= Condiciones de y evaluacion salud adecuadas (está bien en ambas o tiene solo
alguna mal).

- Percepción de vejez: mide la manera en que los adultos mayores enfrentan la vida, ya sea de manera entusiasta o por el contrario resignándose 0 teniendo poco interés por lo que vendrá

- Relaciones sociales: combina dos índices. El primero es una escala de percepciones sobre la calidad de las relaciones familiares que prosentido contrario) las siguientes preguntas: "siente que los miembros de su familia pueden confiar en que se ayudarán para lo que sea", "siente que sus familiares lo toman poco en cuenta", "siente que algunos de sus familiares abusan de Ud.", "siente que en su familia son muy unidos", "siente que sus familiares no lo entienden", "siente que en su familia hay bastantes peleas y desavenencias" (1. sí a meny bast 2 a punas veces, 3 nunencias" (1. si a menudo, 2 algunas veces, 3 nunca). indice va de 1 (malas relaciones familiares) a 3 (excelentes relaciones). Como en la muestra se observaron muy buenas relaciones familiares, e corte se hizo en un punto bastante arriba: 2,5 o más se consideran percepciones positivas. E segundo índice suma la presencia de relaciones calificadas como satistactorias o poco satisfac calife con alguna persona con quien vive o con sus hijos. Combinando estos dos indices, so con- sidera que una persona tiene buenas relaciones familiares cuando tiene percepciones positivas acerca del funcionamiento familiar y no tiene ninguna relación disturbadora.

\section{Medición y procedimientos estadísticos} Luego de la consolidación de las variables a utilizar, se calcularon asociaciones bivariadas entre cada uno de estos predictores y la participación social, todas las cuales son significativas a un nivel de confianza de 95\%, lo cual justifica su inclusión en el modelo, con excepción de la variable 'tiene pareja'. No existen diferencias significativas entre los adultos mayores con pareja y sin pareja y su nivel de participación, por lo cual se decidió excluir esta variable de los modelos

Asimismo, se estimaron correlaciones entre las variables predictoras. Cómo es lógico de pensar, la capacidad funcional se correlaciona fuertemente con las condiciones de salud de la persona, por lo cual se optó por incluir solo una de estas variables en el modelo de regresión. Con respecto al resto de las asociaciones, en general no son demasiado altas, por lo que es probable que tengan efectos independientes sobre la participación social de las personas mayores.

Por último se realizaron estimaciones de modelos de regresión logística binaria por bloques para cada una de las variables de participación social. En el bloque 1 se incluyeron las variables sociodemográficas sexo, edad, región más educación. En el bloque 2 ingresaron las variables predictoras capacidad funcional y percepción de la vejez, para finalmente en el bloque 3 incluir la variable de re-
laciones sociales.

\section{Resultados}

Este estudio considera las respuestas de los 1616 adultos de 60 o más años, de los cuales 918 son mujeres (57\%), edad promedio de 70,7 años -con una desviación estándar de 7,6 años-, con un 28,6\% en el tramo de 75 o más años. Un 54\% vive en regiones y el 46\% restante en la región Metropolitana. Un 7\% no tiene educación, 53\% tiene educación básica, un 27\% educación media y un 13\% educación superior (Cuadro 1)
CUADRO 1.

CAS SOCIODEMOGRÁFICA MUESTRA

\begin{tabular}{|l|l|c|}
\hline \multicolumn{2}{|c|}{ Variable } & Porcentaje \\
\hline \multirow{2}{*}{ Sexo } & Hombre & $43,2 \%$ \\
\cline { 2 - 3 } & Mujer & $56,8 \%$ \\
\hline \multirow{2}{*}{ Edad } & $60-74$ años & $71,4 \%$ \\
\cline { 2 - 3 } & 75 y más & $28,6 \%$ \\
\hline \multirow{2}{*}{ Región } & Metropolitana & $45,8 \%$ \\
\cline { 2 - 3 } & Resto regiones & $54,2 \%$ \\
\hline \multirow{4}{*}{ Educación } & Sin educación & $6,9 \%$ \\
\cline { 2 - 3 } & Educación Básica & $52,8 \%$ \\
\cline { 2 - 3 } & Educación Media & $26,5 \%$ \\
\cline { 2 - 3 } & Educación Superior & $13,4 \%$ \\
\hline \multicolumn{2}{|c|}{ Total muestra } & $\mathbf{1 . 6 1 6}$ \\
\hline
\end{tabular}

Elaboración propia. Base ponderada.

Los resultados para los diversos modelos de regresión estimados se describen en el Cuadro 2. Estos son en parte coincidentes con la literatura expuesta. A modo de resumen se puede apreciar que se confirma la hipótesis de que las mujeres participan más que los hombres, en especial en las actividades religiosas y talleres, donde la chance de participar de las mujeres es cerca de 2 a 3 veces más que la de los hombres, manteniendo el resto de las variables constantes.

También se muestra la relevancia de la educación para la realización de diversas actividades sociales, dado que las personas más educadas poseen mayor probabilidad de participar que las con menos educación. De esta forma los adultos mayores con (a) participan más, especialmente en talleres, donde se puede especular que se requiere de una cierta destreza y de ciertas habilidades que quizás las personas con ciertas limitaciones funcionales pueden no presentar, lo cual reduce la probabilidad de participar en este tipo de actividades sociales.

Junto con lo señalado, la percepción sobre la vejez es una variable tan importante como las mencionadas para explicar la mayor participación por parte de los mayores. El poseer sentimientos positivos sobre la vejez y sentirse con entusiasmo para enfrentar esta etapa, aumenta las chances de participar y de involucrarse de los mayores, opuesto a quienes ven a la vejez con resignación. Se debe mencionar mercepción sobre la vejez es más frecuente entre las personas más educadas, en la medida en que la educación provee de una serie de habilidades psicosociales que ayudan a las personas a enfrentar de mejor modo los nuevos sucesos y pérdidas que ocurren al envejecer, y por tanto ayuda a disminuir las percepciones y sentimientos negativos de esta etapa.

El factor contextual también aparece como un elemento significativo de considerar, en la medida en que las personas mayores que viven en la Región Metropolitana participan menos que las que viven en regiones. Esto en parte puede explicarse por la mayor extensión de la vida en comunidad asociada a las regiones, que puede facilitar la inclusión de los mayores a diversos grupos. En las regiones parece existir mayor contacto entre vecinos, amigos y entre la comunidad en general, lo cual sin duda favorecerá la participación social de los mayores.

Destaca además que la edad no es una variable significativa en los modelos. Este hallazgo permite reafirmar la idea de que la edad no es impedimento en sí mismo para que los mayores participen y sean actores comprometidos. Todo adulto mayor, independiente de su edad, puede estar en condiciones de involucrarse socialmente. Por tanto, más que la edad en sí misma, son otras condicionantes las que limitan la posibilidad de participación entre las personas mayores.

Por último, se encuentra que las características del entorno social presentan un comportamiento ambiguo. Para el nivel de participación general, y para la participación en clubes y talleres, la variable no es significativa. No obstante, para el caso del voluntariado y la religión sí lo es, pero de modo opuesto: las personas con malas relaciones tendrán más probabilidad de participar en voluntariado mientras que las con mejores relaciones tendrán más chance de pertenecer a algún movimiento religioso. Como conjetura puede pensarse que las personas que participan en el voluntariado pueden intentar supli alguna carencia de tipo familiar, buscando así a través del voluntariado lograr establecer relaciones de ayuda y reciprocidad, que en su entorno cercano no consiguen. 


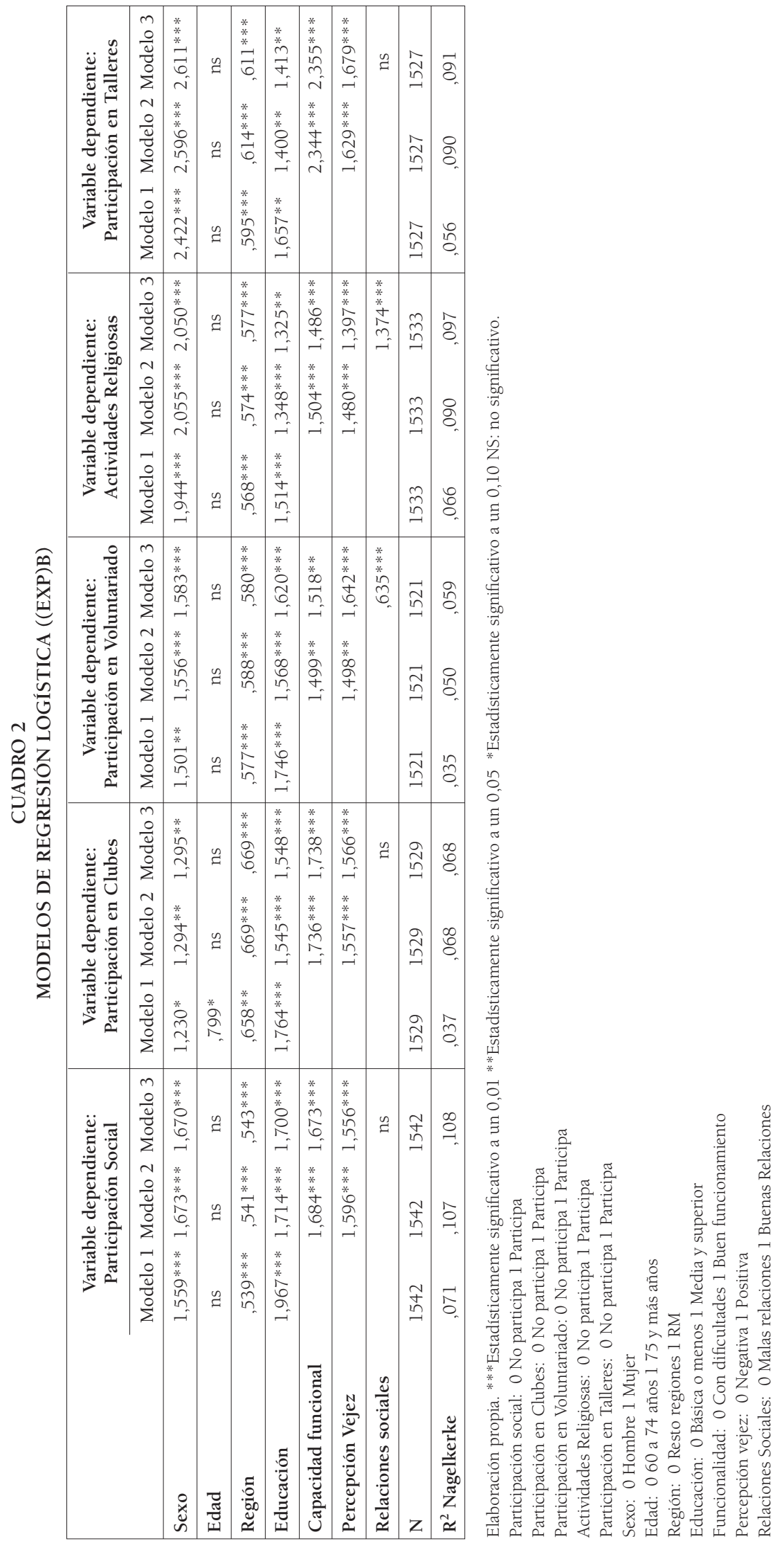

Conclusiones. Limitaciones y alcances

La principal intención de este artículo era conocer los principales factores que explican una mayor probabilidad de involucramiento en la comunidad por parte de los adultos mayores.

En términos generales, más allá de las características sociodemográficas y contextuales, tres son los grandes factores que pueden marcar la diferencia a la hora de participar. El primero dice relación con la educación, la cual no solo es importante porque provee de mejores ingresos y favorece mejores condiciones de salud, que son elementos necesarios para poder participar activamente y mantenerse integrados a la sociedad, sino porque además ayuda a enfrentar de modo más esperanzador la vejez; la educación ayuda a las personas a adaptarse de mejor manera a los nuevos escenarios, favoreciendo con ello una percepción positiva de esta nueva etapa, lo cual es el segundo elemento relevante para una mayor participación. Las personas con una percepción optimista y entusiasta sobre la vejez estarán más motivadas para involucrarse en nuevos proyectos que les ayuden a dar un nuevo sentido a su existencia. Por último, las condiciones funcionales son el otro factor que repercute de modo significativo en las posibilidades de cooperación y de involucramiento de los mayores. Lo interesante de todos estos elementos es que mediante diversos programas gerontológicos pueden lograrse mejoras, que redunden finalmente en una mayor participación social por parte de la población mayor.

Sin duda, todos estos hallazgos vienen a reafirmar una serie de aspectos ya mostrados por la evidencia previa, aún cuando se puede vislumbrar que todavía queda mucho por avanzar Este ha sido un estudio de carácter más bien exploratorio, con lo cual quedan muchos desafios pendientes. Se necesita seguir profundizando y reflexionando sobre la nueva evidencia internacional y nacional, que pueda ayudar a mejorar el modelo contrastado en la presente ocasión, sobre los factores vinculados a una mayor participacion social, introduciendo nuevas vabes y pudiendo además medir otras formas más específicas de participación social, que no pudieron ser cubiertas en la presente ocasión.

\section{Bibliografía}

AGRESTI, A. (2007). An introduction to categorical data analysis. New Jersey: Wiley-Interscience

BARROS, C., FORTTES, A. y AVENDANOO, C. (2004) Componentes de la calidad de vida del adulto mayor y factores asociados. Santiago de Chile: Informe técnico Fondecyt 1020643 .

BARROS, C., FORTTES, A. y HERRERA, S. (2006) Predictores de un buen envejecimiento entre los adultos mayores afiliados a la Caja de Compensación de Los Andes. Santiago de Chile: Universidad Católica - Caja Los Andes.

BECK, S. H. y PAGE, J. W. (1988). Involvement in activities and the psycho-logical well-being of retired men Activities, Adaptation, and Aging, 11(1), 31-47.

CELADE. (2003). Protección y participación en la vejez: escenarios futuros y políticas públicas para enfrentar el envejecimiento en Chile. Santiago de Chile: CEPAL

CEPAL. (2009). Proyecciones de la problación. Santiago de Chile.

CONNIDIS, I. y MCMULLIN, J. (1992). Getting out the house: the effect of childlessness on social participation and companionship in later life. Canadian Journal on Aging, 11(4), 370-386.

DÁVILA DE LEÓN, M. y díAZ MORALES, J. (2009). Voluntariado y tercera edad. Revista Anales de Psicología, 25(2), 375-389.

ENGLER, T. (2005). El empoderamiento de los adultos mayores organizados en la búsqueda de un nuevo contrato social: experiencias del BID y la Red Tiempos. Revista Panamericana Salud Pública, 17(5), 438-443.

GONZÁLEZ, E. (1994). Prevención comunitaria. Revista sobre igualdad y calidad de vida, 25(1), 77-86.

GRIFFIN, J. Y MCKENNA, K. (1998). Influences on leisure and life satisfaction of elderly people. Physical an Occupational Therapy in Geriatrics, 15(4), 1-16.

HOLT-LUNSTAD, J., SMITH, T. y LAYTON, J. (2010) Social Relationships and Mortality Risk: A Metaanalytic. PLos Medicine, 7(7), 1-20.

HOUSE, J. S., LANDIS, K. R. y UMBERSON, D. (1988). Social relationships and health. Science, 241, 540-545.

IMSERSO. (2008). La participación social de las personas mayores. Madrid: Gobierno de España.

KRZEMIEN, D. (2001, Noviembre 2001,). Calidad de Vida y Participacion Social en la Vejez Femenina. Ponencia presentada en la IV Reunión de Antropología del Mercosur, Curitiba, Brasil.

LEFRANCHOIS, R., LECLERC, G. y POULIN, N. (1998). Predictors of activity involvement among older adults. Activities, Adaptation, and Aging, 22(4), 15-29. 
LENNARTSSON, C. y SILVERSTEIN, M. (2001). Does engagement with the life enhance survival of elderl people in Sweden? Journal of Gerontology, 56b(6) 335-342

MCDONALD, J. (1996). Community participation in an Australia retirement village. Australian Journal on Ageing, 25(1), 167-171

MCGUINN, K. y MOSHER, A. (2000). Participation in recreational activities and its effect on perception of life atisfaction on residential settings. ion, and Aging, 25(1), 77-86

ROWE, J. W. y KAHN, R. L. (1997). Successful aging. The Gerontologist, 37, 433-440.

SEEMAN, T. (2000). Health promoting effects of friends comes in older adult. American Journal Health Promotion, 14(6), 362-370.

SENAMA. (2003). Chile necesita a sus Adultos Mayores: Participación social. Santiago de Chile: Servicio Nacional del Adulto Mayor

TRIADÓ, C., CELDRÁN, M., CONDE, L. y OTROS (2008). Envejecimiento productivo: la provisión de cuidados de los abuelos a los nietos. Implicaciones

VEGA, J., BUZ, J. y BUENO, B. (2002). Niveles de actividad y participación social en las personas mayores de 60. Revista Interuniversitaria de Formación de Profesorado, 45 .

WARBURTON, J., FERRY, D. J., ROSENMAN, L. S. y OTROS (2001). Differences between older volunteers and nonvolunteers. Research on Aging, 23(5), 586605.

WARBURTON, J. y STIRLING, C. (2007). Factors affecting volunteering among older rural and city dwelling adults in Australia. Educational Gerontology, 33(1), 23-43.

\section{Gestión del deseo en la intervención social: claves estéticas y el riesgo de la participación}

The management of desire in Social practice: Aesthetic key and the Risk of participation

JaVIERA ROA INFANTE

Magister en Trabajo Social, PUC. Marín No30, Santiago Centro. correo electrónico: jiroa@uc.cl.

\section{Resumen}

Existe un creciente interés desde las politicas sociales por incorporar al otro (usuario, beneficiario poblador, etc.) en la intervención social: su opinión, inquietudes, intereses. Muchas veces, esta intención aparece bajo la categoria de participación, la que intenta ser instalada como modo de hacer en procesos desplegados por el Estado, por privados y su RSE, por gobiernos locales y ONGs Este documento muestra cómo estas buenas intenciones participativas se vuelven peligrosas, si es que no asumen el desafio de incorporar al otro en su complejidad, generándose muchas veces respuestas anestesiadas, que no hacen más que reducir o anular su condición de sujeto. De esta formestas anestesiadas, que no hacen más que reducir o anular su condición de sujeto. De esta desde la critica a la participación se propone como ejemplo para abrir la categoria del deseo nerando nuevas posibilidades de trabajo, a través de lo que hemos denominado la "gestión de deseo en la intervención social.

Palabras clave. Participación, estética, intervención social, sujeto, deseo.

\section{Abstract}

There is a growing interest from social policy to incorporate the other (user, beneficiary, resident, etc.) in social practice: its view, concerns, interests. Many times, this intention appears under the category of participation, which is intended to be installed as a way of doing in processes undertaken by the State, by private and their CSR, local governments and NGOs.

This document will show how these participatory good intentions become dangerous, if it is not assumed the challenge of incorporating the other in their complexity, often generating responses anesthetized, which only serve to reduce or denie their status as subjects.

Thus, the criticism to participation is proposed as an example to open a category of desire from the aesthetic dimension of social practice -negative rational display- generating new employ-

ment opportunities, through what we will call the "management desire for social practice".

Key words. participation aesthrtic, social practice, subject, desire.

Los riesgos de la participación

Cada vez más se exige a quienes implementa diseña políticas sociales y procesos de intervención social incorporar a los afectados en la solución de sus problemas. Muchas veces este asunto se resuelve con el requerimiento de hacer participar a la gente. Presupuestos participativos, consultas ciudadanas, mesas de trabajo, son algunas de las metodologías que se despliegan en estos términos, dibujándose una linea de tendencia de las policies, fortalecida por un amplio consenso a nivel nacional respecto de la importancia de la participación social de cara al fortalecimiento de la ciudadania, la democracia el ejercicio de las políticas públicas (Serrano, 1998:1)

¿Por qué esto puede ser riesgoso? Si más allá de la retórica participativa algún modelo de intervención logra hacer efectivamente parte al usuario en el proceso, ¿cómo podría ser en alguna medida problemático? Y es que incorporar al otro en la intervención social enfrenta el desafío de la complejidad del otro. No es posible su resolución con mecanismos lineales y unidimensionales, porque 